\title{
Child Care Practices and Associated Factors among Women of Childbearing Age Attending Health Facilities in Dekina, North-Central, Nigeria
}

\author{
Olaoluwa Samson Agbaje ${ }^{1, ~ *}$, Benedicta Ndidi Agu ${ }^{1}$, Prince Christian Ifeanachor Umoke ${ }^{2}$, \\ Felicia Saturday Ekpu ${ }^{1,3}$, Nkiruka Hope Orungbe ${ }^{1}$ \\ ${ }^{1}$ Department of Public Health, Faculty of Health Sciences, Madonna University, Elele, Nigeria \\ ${ }^{2}$ Department of Human Kinetics and Health Education, University of Nigeria, Nsukka, Nigeria \\ ${ }^{3}$ Department of Physical and Health Education, University of Uyo, Uyo, Nigeria
}

Email address:

agbajesam@yahoo.com (O. S. Agbaje)

${ }^{*}$ Corresponding author

\section{To cite this article:}

Olaoluwa Samson Agbaje, Benedicta Ndidi Agu, Prince Christian Ifeanachor Umoke, Felicia Saturday Ekpu, Nkiruka Hope Orungbe. Child Care Practices and Associated Factors among Women of Childbearing Age Attending Health Facilities in Dekina, North-Central, Nigeria. Science Journal of Public Health. Vol. 4, No. 5, 2016, pp. 366-374. doi: 10.11648/j.sjph.20160405.11

Received: July 18, 2016; Accepted: July 26, 2016; Published: August 15, 2016

\begin{abstract}
Child care practices have contributed immensely to decreased child mortality especially in developing nations. Investigation of child care practices and associated factors among women is necessary to provide insight into extent of adoption of maternal, newborn and child health strategies and suggest enhancement strategies where possible. This examines child care practices and associated factors among women in Dekina LGA, Kogi State. Cross-sectional survey research design was adopted for the study. The population of the study comprised 971 women of childbearing age accessing health services at health facilities in Dekina LGA, Kogi State, North-Central Nigeria. A sample of 384 women of child bearing age was selected through multi-stage sampling procedure. Well-completed 299 copies of the researcher-designed Women's Child Care Practices and Associated Factors Questionnaire (WCCPAFQ), which comprised sections A, B and C was used for data collection. The reliability co-efficient of the instrument was determined using Split-half method and Spearman-Brown Correction Formula. The reliability co-efficient of the instrument was 0.65. Frequency and percentage, Spearman's $p(r h o)$ rank Correlation Coefficients and logistic regressions were employed for data analyses. A logistic regression was run to investigate the relationship between the dependent and independent variables. Results showed that women moderately practised (57.5\%), $51.9 \%$ of women perceived that the outlined factors affected child care practices while there was low relationship between outlined factors and child care practices among women (maternal age $r=0.11, \mathrm{p}<0.05$; maternal education $r=0.10, \mathrm{p}<$ 0.05 ). Independent variables of age $[\mathrm{OR}=1.65,95 \%$ (C. I: $0.56-4.88)]$ and maternal education $[\mathrm{OR}=2.45,95 \%$ (C. I: 1.01 5.94)] had significant influence on child health care practices. It was recommended that government; private organizations, Non-governmental Organizations (NGOs) and other stakeholders in the health sector should implement holistic programmes that will enhance women's capacity to effectively and efficiently adopt integrated maternal, newborn child health survival strategies for quality child care practices. The general public should also be enlightened via health education programmes on the associated health risks of poor child care practices prevalent in many rural communities.
\end{abstract}

Keywords: Child Care Practice, Associated Factors, Psychosocial Development, Exclusive Breastfeeding

\section{Introduction}

Globally, child care practices have contributed immensely to decreased child mortality. Although, literature differs on the basis of countries studied, approximately 6.6 million children worldwide, that is, 18,000 children per day died before reaching their fifth birthday [1]. The leading causes of death among children aged less than five years include: 
pneumonia, prematurity, birth asphyxia, diarrhea and malaria. About $45 \%$ of under-five deaths are linked to undernutrition [1]. In spite of the significant progress made in recent years to reduce under-five mortality, significant inequities between and within countries continue to exist. These are not only driven by poverty, but are intrinsically linked to social exclusion and de jure and de facto discrimination. Therefore, efforts to eliminate under-five mortality require a comprehensive and holistic approach, which explicitly recognizes and integrates relevant human rights [1].

Nigeria is the second largest contributor to under-five and maternal mortality rates in the world. Underneath the statistics lies the pain of human tragedy, for thousands of families who have lost their children. Even more devastating is the knowledge that, according to recent studies, essential interventions reaching women and babies on time would have averted most of these deaths. Although, analyses of recent trends show that the country is making progress in cutting down infant and under-five mortality rates, the pace still remains too slow to achieve the Millennium Development Goals (MDGs) of reducing child mortality by a third by 2015 [2]. It has been observed that Nigeria is off track in meeting MDG 4 and according to the United Nations mortality estimates, Nigeria has only achieved an average of $1.2 \%$ annual reduction in under-five mortality since 1990. And in order to meet MDG 4, Nigeria needed to have achieved an annual reduction rate of $10 \%$ in the five years leading to 2015 [3-4, 5-7]. Preventable or treatable infectious diseases such as malaria, pneumonia, diarrhea, measles and HIV/AIDS account for more than 70 per cent of the estimated one million under-five deaths in Nigeria [8]. This unpleasant situation hampers child development in Nigeria with attendant consequences for a fulfilled adult life.

Children are vital to the nation's present and its future. Parents, grandparents, aunts, and uncles are usually committed to providing every advantage possible to the children in their families, and to ensuring that they are healthy and have the opportunities that they need to fulfill their potentials in life. Yet communities vary considerably in their commitment to the collective health of children and in the resources that they make available to meet children's needs. This is reflected in the ways in which communities address their collective commitment to children, specifically to their health [9]. Child health is the purview of pediatrics, which became a medical specialty in the mid-nineteenth century. Before that time, the care and treatment of childhood diseases were included within such areas as general medicine, obstetrics, and midwifery [10].

Healthy children live in families, environments, and communities that provide them with the opportunity to reach their fullest developmental potential. Current conceptions of children's health have evolved and expanded significantly from early notions of health as merely a state of being free of disease. National Research Council and Institute of Medicine defined children's health as the extent to which individual children or groups of children are able or enabled to develop and realize their potential; satisfy their needs; and develop the capacities that allow them to interact successfully with their biological, physical, and social environments [11]. This change in definition parallels the expansion of concerns in the practices of individual children's health care professionals. This broader definition of children's health acknowledges the influences of the biological, behavioural, social, and physical environments on health trajectories.

Child care practices have been variously conceptualized in literature. Child care practices refer to the quality, quantity, diversity and availability of health care services given to a child which must not be necessary from the health services providers and parents but from other older adults [12]. WHO defined child care practice as the physical, social, and health care given to a child [1]. Child care practices are always part of a system of care that covers the whole cycle of childbearing and childrearing in the family. This can also be achieved by participation from the community level. It is carried out by community health workers who live in the community and are properly trained to carry out the essential services which the community needs and can afford. Child care practices include: exclusive breastfeeding or other forms of breast feeding, complementary feeding, hygiene, immunizations, micronutrients, use of bed nets for malaria prevention, psychosocial development, compliance with medical/health advice, antenatal care, home care for illness, home treatment of minor infections and care seeking practices $[2,13]$.

Child care has been classified into three types, namely: centers, non-relative, or relative care [14]. A center refers to a group setting designed for the care of young children. It includes programmes designed primarily for enrichment or early education (i.e., head start, preschools, or after-school programmes), as well as settings designed primarily to provide care while parents are working. Non-relative care can occur in the caregiver's home (e.g., family child care homes) or in the child's home. Parents typically pay for such care. Some such child care homes are licensed, certified, or registered; some receive training and technical assistance, but many do not. Relative care is provided by grandparents, siblings, or other relatives in the child's home or in their own homes $[14,15]$. This study restricted its scope to relative care which is the commonest form of child care in many communities in Nigeria including Dekina LGA, Kogi State. WHO reported that certain factors influence child care practices especially in developing nations including Nigeria [2]. These factors can be social factors, familial factors, maternal factors, and environmental or cultural factors.

In this study, social and familial factors were studied because they exert more profound effects on child care practices among people in diverse climes. The term social factor refers to the facts and experiences that influence or control individuals' personality, attitudes and lifestyle. These factors help an individual live well in harmony with others in the society. Young children can be affected by many social, economic and environmental factors both in positive and negative ways. Because children are so vulnerable, they can be easily affected by things many parents and adults take for 
granted, according to [16]. Social factors that affect child health practices include learning environment, parental interaction, improper speech and language development, preconception health status, age, access to appropriate preconception and inter-conception health care, racial and ethnic disparities in infant mortality, family income, educational attainment among household members and health insurance coverage. Children reared in safe and nurturing families and neighborhoods are likely to have better outcomes as adults [2].

Familial factors refer to elements within a family which account for a condition [17]. These familial factors include changes in families-structural and cultural diversity, changes in family circumstances-employment, and changes in family circumstances-social and emotional. Families have become more diverse in their structure and background and there are more families with multiple needs making parenting more stressful business for many families [2, 18]. The negative interactions of these factors (social and familial) can precipitate a spectrum of unwholesome traits such low selfesteem, abnormal behaviours, mental retardation and other developmental anomalies which make children become burdens to the society and immediate families. However, when children are reared in safe, nurturing families and neighborhoods they are likely to have better outcomes as adults.

Demand for effective child care has increased markedly in proportion to the number of mothers with young children employed outside the home. As society has become increasingly mobile, with fewer extended families living in close proximity to one another, many working mothers cannot rely on relatives to provide child care. Children are vulnerable to certain types of deprivation; even short periods of deprivation can impact their long term development. Children living in poverty experience deprivation of the material, spiritual, and emotional resources needed to survive, develop and thrive, leaving them unable to enjoy their rights, achieve their full potential or participate as full and equal members of society [18]. If children do not receive adequate care such as nutrition, they grow smaller in size and intellectual capacity, are more vulnerable to life-threatening diseases, perform worse in school, and ultimately, are less likely to be productive adults. Child poverty threatens not only the individual child, but is likely to be passed on to future generations, entrenching and even exacerbating inequality in society [19].

This study is based on two theories, namely: socioecological model and symbolic interaction. The social ecological model (SEM) helps to understand factors affecting behaviour and also provides guidance for developing successful programmes through social environments. Social ecological models emphasize multiple levels of influence (such as individual, interpersonal, organizational, community and public policy) and the idea that behaviours both shape and are shaped by the social environment. The principles of social ecological models are consistent with social cognitive theory concepts which suggest that creating an environment conducive to change is important to making it easier to adopt healthy behaviours such as child care practices. While SEM provides a framework for considering factors influencing child care practices; symbolic interactionism provides a theory for considering the subjective inner processes that mothers use in making their choices and deciding on the significance of their actions.

The development of the self is central to symbolic interactionism. This happens as an individual imaginatively constructs the attitudes of the other about a particular role, and thus anticipates the behaviour of the other. Not all "others" are equally influential in this construction process. The 'generalized other', in the most abstract sense, is the view of relevant rules and roles of society as a whole. In the case of child health practices, marketing and media portrayals of infant or child care are influential [20]. 'Reference groups' are social groups with which people identify that are capable of influencing them. Family, friends, peers, neighborhood, and workplace groups may become such reference groups for mothers who are feeding their infants [21]. 'Significant others' are considered the most influential person or group of persons with whom an individual interacts. Child care practices can be framed in symbolic interaction terms. Individual mothers must decide whether they will take on the roles of child feeding, home illness management, hygiene practices and mother-child relationship development or even all. A child health practice is therefore can be explained in symbolic interactionist's terms.

Children face numerous environmental and social challenges in the contexts of families, schools, and communities that significantly affect their well-being and health outcomes. Unhealthy outcomes for children contribute to a negative health trajectory over time. Poor child health and developmental outcomes contribute to poorer short- and long-term growth and development outcomes for children, some of which have lifelong impacts. Poor child health and developmental outcomes result in increased cost and strain to families and to the larger society. These costs can include costs associated with health care, education, justice system, non-profit organizations and all levels of government [22]. To address this public health problem, adequate observance of child care practices, which is integral part of maternal, newborn and child health $(\mathrm{MNCH})$ strategy by mothers and identification of associated factors that could promote or negate child care practices are vital determinants of children's health and well-being. However, there is paucity of information on child care practices and associated factors among mothers in Dekina LGA. Hence, this study sought to ascertain child care practices and associated factors among mothers attending health facilities in Dekina LGA, Kogi State, North-central Nigeria

\section{Purpose of the Study}

The main purpose of this study was to determine child care practices (CCPs) and associated factors among women of 
childbearing age attending health facilities (HFs) in Dekina LGA, Kogi State. Specifically, the study sought to determine:

i. Proportion of women attending health facilities that adopted child CCPs in Dekina LGA;

ii. Determine the factors associated with CCPs among women attending HFs in Dekina LGA;

iii. Relationship between maternal age and CCPs among women attending HFs in Dekina LGA;

iv. Relationship between level of education and CCPs among women attending HFs in Dekina LGA;

v. Based on the study objectives the following null hypothesis was formulated:

vi. There is no significant relationship between selected demographic factors (age and level of education) and CCPs among women attending HFs in Dekina LGA.

\section{Methods and Materials}

The cross-sectional survey research design was adopted for the study. The population for study consisted of 971 women who registered for ANCs at the PHCs between January and March, 2014 constituted the study population. Dekina LGA has ten political wards namely: Iyale, Anyigba, Odu 2, Dekina, Abocho, Oganeenugu, Ogbabede, Okura, Ojikpadala, Odu 1, Emewe, and Egume. About 110 government-owned health facilities are distributed across the villages in different communities. The sample size was determined using Fisher's (Vaughan \& Morrow, 1989) sample size determination formula. The formula and calculation of the sample size are illustrated below:

$$
\mathrm{ME}=z \sqrt{ } \frac{p(1-p)}{n}
$$

Where:

$\mathrm{ME}$ is the desired margin of error

$\mathrm{z}$ is the $\mathrm{z}$-score, i.e. 0.05 for a $95 \%$ confidence interval

$\mathrm{p}$ is our prior judgment of the correct value of prevalence of expected attribute/characteristic (50\% of women expected to have at least performed one child care practice)

$\mathrm{n}$ is the required sample size

So in this case we set ME equal to $0.05, z=1.96$ and $\mathrm{p}=$ 0.5 , and $\mathrm{n}$ becomes

$$
\begin{aligned}
& 0.05=1.96 \sqrt{ } 0.5(1-0.5) / \mathrm{n} \\
& 0.5 \times 0.5 / \mathrm{n}=(0.05 / 1.96)^{2} \\
& 0.25 / \mathrm{n}=0.00065 \\
& \mathrm{n}=0.25 / 0.00065=384
\end{aligned}
$$

Thus, a sample of 384 women attending health facilities (HFs) in Dekina LGA was used for the study. The multistage sampling procedure was adopted to draw the sample for the study. The first stage involved use of simple random sampling technique of balloting without replacement to select five political wards out of the existing 12 political wards. The second stage involved the use of sample random sampling technique of balloting without replacement to select four health facilities in each of the five sampled political wards. This procedure yielded a total of 20 HFs. Furthermore, convenience or purposive sampling technique was used to select 19 women of childbearing age in each of the three sampled primary health centers/health facilities. This process produced a total of 380 women. However, four women were randomly selected to complete the sample. At the end of these procedures, a total of 384 women were selected.

\subsection{Research Instrument}

A-25 item researcher-designed on Women's Child Care Practices and Associated Factors Questionnaire (WCCPAFQ) was utilized for data collection. It comprised three sections, namely: A, B, and C. Section A elicited data on basic demographic information of respondents (age and level of education). Section $\mathrm{B}$ had 15 questions, which elicited information on practice of child care while Section $C$ had eight questions that elicited information on factors affecting child care practices. Three experts validated the instrument. The response option for sections $\mathrm{B}$ and $\mathrm{C}$ was a dichotomous response format of 'Yes' or 'No'. Each of the experts was given a drafted copy of the questionnaire and accompanied with specific purposes of the study, research questions and hypotheses. Split-half method of reliability testing was used to determine reliability index of WCCPAFQ while Spearman Brown Correction Statistic was employed to establish reliability co-efficient of the instrument. The reliability coefficient value of 0.65 was obtained.

\subsection{Method of Data Collection}

In other to gain access to the MOs and reach the respondents, a letter of introduction was collected from the Head, Department of Public Health Madonna University Elele, Rivers State explaining the purpose of the study. This was submitted to the head of each sampled health facilities. The researchers and nurses who served as research assistants (RAs) administered the copies of the questionnaire to participants. Informed consent was sought before administration of copies of questionnaire. In all, 384 copies of the questionnaire were distributed by the researchers and research assistants to the participants. The researchers and their research assistants pleaded with the subjects to complete copies of the questionnaire given to them on the spot and return immediately, to ensure high return rate. All 384 copies of the questionnaire were retrieved after completion. The inclusion criteria included being a woman who registered for any of the $\mathrm{MNCH}$ strategies or programmes between January-March, 2014 in the selected health facilities and was willing to participate in the study after given informed consent. The exclusion criteria included not willing to participate in the study and/or inappropriate or inconsistent response to the questionnaire items based on the investigators' discretion.

\subsection{Method of Data Analysis}

Out of the 384 copies of WCCPAFQ distributed and retrieved, 299 copies were used for data analysis. Data were analyzed using Statistical Package for Social Sciences batch system (International Business Machines Version 20). The 
analysis involved a number of stages. The first stage was a descriptive analysis to examine the characteristics of the sample and estimate child care practices (CCPs) observed by women based on selected socio-demographic factors (maternal age and level of education). Frequency and percentage and Spearman's p (rho) rank correlation coefficients were used for analyzing data generated via sections B and C. Borg's guidelines for interpretation of correlation coefficients were employed for interpreting computed correlation coefficient values. Borg's guidelines assume that correlations relate to a hundred or more subjects [23]. Thus, a correlation value within the range of $0.20-0.35$ was interpreted as low relationship; a correlation value within the range of $0.35-0.65$ was interpreted as moderate relationship; a correlation value within the range of $0.65-0.85$ was interpreted as strong relationship; and a correlation value $\geq 0.85$ was interpreted as very strong relationship.

The second stage was to conduct bivariate analysis of the associations between socio-demographic factors, and the outcome variables $(\mathrm{CCPs})$. Only significant factors $(p<0.05)$ or those that were considered significant were used in the multivariate analysis. We used logistic regression to conduct the multivariate analysis because the outcome variable is dichotomous. A total of two regression models were constructed. The first model contains demographic factors of women (maternal age and education level). In the second model, child care practices were introduced. Association was considered statistically significant at $P<0.05$ and the estimated coefficients (b) when exponentiated were interpreted as the odds of use of CCPs and non-use of CCPs in the reference group. The logistic regression model was used to examine the relationship (odds ratios) between the dependent variable and a set of pre-selected independent variables. All analyses were adjusted for design effects (sample weight, strata, and cluster). This was conducted using SPSS Complex Samples package.

\section{Ethical Consideration}

Informed consent was sought before interviewing each respondent. Participants were assured of confidentiality, privacy and anonymity of information provided. It was explained to the participants that the confidentiality of information provided in the questionnaire would be guaranteed and treated as confidential. It was also made clear to the participants that took part in the study that participation was voluntary, and that those who chose to participate were free to withdraw their participation at any stage if they so wished.

\section{Results and Discussion}

This section presents results from the univariate (Tables 1 and 2) bivariate (Tables 3, 4 and 5) and multivariate (Table 6) analyses. The results (for both the bivariate and multivariate analysis) are based on the $95 \%$ confidence interval.

Table 1. Distribution of Selected Demographic Characteristics of WCBA (15-49 years) attending Health Facilities in Dekina LGA.

\begin{tabular}{lll}
\hline Variables & $\boldsymbol{n}$ & $\boldsymbol{\%}$ \\
\hline Maternal Age & & \\
\hline 15-24 years & 90 & 30.1 \\
25-34 years & 122 & 40.8 \\
35-44 years & 59 & 19.7 \\
$\geq 45$ years & 28 & 9.4 \\
Maternal Level of Education & & \\
No Formal Education & 39 & 13.1 \\
Primary Education & 59 & 19.7 \\
Secondary Education & 81 & 27.1 \\
Tertiary Education & 120 & 40.1 \\
\hline
\end{tabular}

Table 2. Proportion of WCBA that Observed Child Care Practices-CCPs $(n=299)$.

\begin{tabular}{|c|c|c|}
\hline Items & Yes $(\%)$ & No $(\%)$ \\
\hline Breastfeed child/children exclusively for up to six months & $207(69.2)$ & $92(30.8)$ \\
\hline Breastfeed child/children exclusively for just three months & $120(40.1)$ & $179(59.5)$ \\
\hline Provide additional micronutrients from supplements to children after six months & $205(68.6)$ & $94(31.4)$ \\
\hline Give child/children complementary foods to prevent iron-deficiency & $149(49.8)$ & $150(50.2)$ \\
\hline Attendance at baby-friendly hospitals to improve EBF practices & $232(77.6)$ & $67(22.4)$ \\
\hline Feed child freshly prepared energy-and nutrient-rich complementary foods regularly & $120(40.1)$ & $179(59.9)$ \\
\hline Immunize child/children through NPI routine immunization and SIAs & $236(78.9)$ & $63(21.1)$ \\
\hline Rarely immunize child/children due to negative health worker attitude & $110(36.8)$ & $189(63.2)$ \\
\hline Give child/children herbal medicine due to inaccessibility to immunization service & $160(53.5)$ & $139(46.5)$ \\
\hline Regular washing of hands with soap and water/cleansing agents after defecation & $205(68.6)$ & $94(31.4)$ \\
\hline Regular hand washing with soap and water/cleansing agents after defecation before preparing child's meal & $208(69.6)$ & $91(30.4)$ \\
\hline Always wash hands after defecation but not after disposing of their child's faeces & $95(31.8)$ & $204(68.2)$ \\
\hline Wash hands with soap and water/cleansing agents before feeding children & $207(69.2)$ & $92(30.8)$ \\
\hline Proper disposal child/children's faeces using water closet or pit latrine & 179 (59.9) & $120(40.1)$ \\
\hline
\end{tabular}

*Percentage scores in parentheses

Key

$0-49 \%=$ Low proportion

$50-79 \%=$ Moderate proportion

$80-100 \%=$ High proportion

Data in Table 2 show that moderate proportion of women observed child care practices $(57.5 \%)$. 
Table 3. Perceived Factors Affecting CCPs WCBA in Dekina LGA $(n=299)$.

\begin{tabular}{|c|c|c|}
\hline Items & Yes $(\%)$ & No $(\%)$ \\
\hline Mother's age at first birth affects her practice of child care & $122(40.8)$ & $177(59.2)$ \\
\hline $\begin{array}{l}\text { Familial circumstances like unemployment and work schedules influence mothers' practice of child care e.g. } \\
\text { exclusive breastfeeding }\end{array}$ & $228(76.3)$ & $71(23.7)$ \\
\hline Poverty or poor household income level is a major determinant of child care & $129(43.1)$ & $170(56.9)$ \\
\hline Family disorganization and lack of social support affect child care practices & $180(60.2)$ & $119(39.8)$ \\
\hline Location of child health facility/clinic hinders effective practice of child care & $124(41.5)$ & $175(58.5)$ \\
\hline Shortage of health care personnel at PHC affects mothers' practice of child care & $138(46.2)$ & $161(53.8)$ \\
\hline Attitude of mothers can also affect child health & $168(56.2)$ & $131(43.8)$ \\
\hline$\%$ Average & 51.9 & 48.1 \\
\hline
\end{tabular}

*Percentage scores in parentheses

Data in Table 3 show that overall, 51.9 per cent of women perceived that the outlined factors affected their child care practices.

Table 4. Relationship between Maternal Age and CCPs among WCBA in Dekina LGA $(n=299)$.

\begin{tabular}{ll}
\hline Items & $r$ \\
\hline Breastfeed child/children exclusively for up to six months & $.353^{* *}$ \\
Breastfeed child/children exclusively for just three months & $-.273^{* *}$ \\
Provide additional micronutrients from supplements to children after six months & $.282^{* *}$ \\
Give child/children complementary foods to prevent iron-deficiency & $-.217 * *$ \\
Attendance at baby-friendly hospitals to improve EBF practices & $-.282^{* *}$ \\
Feed child freshly prepared energy-and nutrient-rich complementary foods regularly & $-.293 * *$ \\
Immunize child/children through NPI routine immunization and SIAs & .036 \\
Immunize child/children when ill or sick & -.053 \\
Rarely immunize child/children due to negative health worker attitude & $.154 * *$ \\
Give child/children herbal medicine due to inaccessibility to immunization service & $.120^{*}$ \\
Regular washing of hands with soap and water/cleansing agents after defecation \\
Regular hand washing with soap and water/cleansing agents after defecation before preparing child's meal \\
Always wash hands after defecation but not after disposing of their child's faeces \\
Wash hands with soap and water/cleansing agents before feeding children \\
$\begin{array}{l}\text { Proper disposal of child/children's faeces using water closet or pit latrine } \\
\text { Overall } r\end{array}$ \\
\hline
\end{tabular}

$* *$ Correlation is significant at the 0.01 level (two tailed)

*Correlation is significant at 0.05 level (two tailed)

Keys for Interpretation of Correlation Coefficients

0.20-0.35 = Low Relationship

0.35-0.65 = Moderate Relationship

$0.65-0.85=$ Strong Relationship

$\geq 0.85$ = Very Strong Relationship

Data in Table 4 indicate that there is low relationship $(r=0.11, p<0.05)$ between maternal age and child care practices.

Table 5. Relationship between Maternal Education and CCPs among WCBA $(n=299)$.

\begin{tabular}{ll}
\hline Items & $r$ \\
\hline Breastfeed child/children exclusively for up to six months & $.382^{* *}$ \\
Breastfeed child/children exclusively for just three months & $-.171^{* *}$ \\
Provide additional micronutrients from supplements to children after six months & -.067 \\
Give child/children complementary foods to prevent iron-deficiency & .110 \\
Attendance at baby-friendly hospitals to improve EBF practices & $.252^{* *}$ \\
Feed child freshly prepared energy-and nutrient-rich complementary foods regularly & $-.626^{* *}$ \\
Immunize child/children through NPI routine immunization and SIAs & $.174^{* *}$ \\
Immunize child/children when ill or sick & $-.178^{* *}$ \\
Rarely immunize child/children due to negative health worker attitude & .063 \\
Give child/children herbal medicine due to inaccessibility to immunization service & $-.215^{* *}$ \\
Regular washing of hands with soap and water/cleansing agents after defecation & $-.313^{* *}$ \\
Regular hand washing with soap and water/cleansing agents after defecation before preparing child's meal & $-.195^{* *}$ \\
Always wash hands after defecation but not after disposing of their child's faeces & $-.312^{* *}$ \\
Wash hands with soap and water/cleansing agents before feeding children & -.037 \\
Proper disposal of child/children's faeces using water closet or pit latrine & .057 \\
\end{tabular}

**Correlation is significant at the 0.01 level (two tailed)

*Correlation is significant at 0.05 level (two tailed) 
Data in Table 5 indicate that there is low relationship ( $r=$ $0.10, p<0.05)$ between maternal education and child care practices.

Table 6. Logistic regression (Odds Ratio) of Factors Associated with CCPS among women in Dekina LGA $(n=299)$.

\begin{tabular}{lll}
\hline \multirow{2}{*}{ Variable } & \multicolumn{1}{l}{ Child Care Practices } & \\
\cline { 2 - 3 } & Univariable effects OR (95\% C. I.) & $\boldsymbol{p}$ \\
\hline Maternal Age & & \\
15-24 years* & 1 & 0.06 \\
25-34 years & $0.55(0.29-1.04)$ & 0.36 \\
35-44 years & $1.65(0.56-4.88)$ & 0.37 \\
245 years & $0.58(0.18-1.89)$ & \\
Maternal Level of Education & \\
No Formal Education* & 1 & 0.21 \\
Primary Education & $1.88(0.69-5.06)$ & 0.2 \\
Secondary Education & $1.79(0.72-4.49)$ & 0.05 \\
Tertiary Education & $2.45(1.01-5.94)$ & 0.39 \\
Constant & 1.49 & \\
\hline
\end{tabular}

Note. $*$ Reference group

**Significant at $\mathrm{p}<0.05$.

$\mathrm{CCPs}=$ Child care Practices

$\mathrm{OR}=$ Odds Ratios

$\mathrm{CI}=$ Confidence Intervals.

Data in Table 6 indicate that pertaining to maternal age, women aged $35-44$ are more likely $[\mathrm{OR}=1.65,95 \%$ (C. I: $0.56-4.88)]$ to use CCPs than those in the reference group (15-24 years) while women aged $\geq 45$ years are more likely $[\mathrm{OR}=0.58,95 \%$ (C. I: $0.18-1.88)]$. Concerning maternal education, women with tertiary education are more likely $[\mathrm{OR}=2.45,95 \%$ (C. I: $1.01-5.94)]$ to use CCPs than those with no formal education. Furthermore, women with secondary education are less likely $[\mathrm{OR}=1.79,95 \%$ (C. I: $0.72-4.49)$ ] to use CCPs than those with primary education where women in the primary, secondary education and tertiary education groups are associated with the use of CCPs at $\mathrm{P}<0.05$.

This study is among the first to use an ecological framework [31] to examine how maternal, neighborhood, and familial factors influence child care practices (CCPs) by mothers. The findings also showed that CCPs, though similar varied across the selected demographic variables. The following discussion considers the most important of these findings, and interprets them more broadly in the context of related previous research. The findings are discussed based on four specific objectives and null hypothesis that guided the study.

Results in Table 2 indicate that women adopted child care practices at moderate level $(57.5 \%)$. The finding is consistent with findings of a study conducted on childrearing practices and their associated beliefs in Nigeria. They reported that women adopted relative childrearing practices in five LGAs in Nigeria [24].

From this study, the factors responsible for average use of CCPs include a diversity of interplaying social, economic and health system factors, which operate at various levels, namely: the household, community, health facilities and larger social environments. The results in Table 3 showed that $51.9 \%$ of women perceived that the outlined factors affected child care practices. This finding is in consonance with the findings of previous studies [16, 25-26] who investigated factors affecting child care options and practices. Furthermore, these studies have examined how factors such as income level, family structure, maternal educational, work schedules and environment affect parental choice of child care practices. Household income generally has been found to be an important factor influencing choice. To further substantiate this finding, Pungello and Kurtz-Costes relied on Bronfenbrenner's concepts in developing a model to explain choice of child care. Their model attempted to elucidate the relationships among the environmental context (e.g., employment constraints, child care availability), maternal beliefs (e.g., beliefs concerning the effects of child care), maternal behaviours (i.e., child care search and selection behaviours), and child outcomes. They asserted a web of factors affect child care practices among care-givers including mothers [25].

Results of bivariate analysis in Table 4 show the relationship between maternal age and observed CCPs. Although, mother's age at first birth is increasing globally due to demographic transition, economic pressure and desire for higher education among women (16), child care practices still vary according to maternal age. Although, influence of age on CCPs among mothers in literature is inconsistent, the finding shows that there was low relationship between maternal age and CCPs. Literature shows that older women (and those with higher parity) tend to use maternal health services less frequently than younger women with fewer children [27]; older women who have given birth before tend to believe that they are better equipped to handle their pregnancy [28], this could explain the minimal use of maternal health services among those aged forty and above and those with more than six children.

The study found that woman's education has a significant influence on CCPs. Results of bivariate analysis in Table 5 show that there was a low relationship between maternal education and CCPs among women. This finding is consistent with findings of authors who reported that mothers with less education (high school diploma only or less) were more likely to rely on relative care than mothers with higher levels of education [29]. This relationship may be rooted in the association between mothers' educational attainment and their weighting of various attributes of child care practices. This finding is also consistent with that of Sika-Bright who conducted a survey on socio-cultural factors influencing infant feeding practices of mothers attending welfare clinic in Cape Coast, Ghana and reported that mothers with higher educational degrees were not exclusively breastfeeding their children [30]. Bick, MacArthur and Lancashire made it clear that working mothers are less likely to maintain breastfeeding. It follows therefore that, educated mothers are mostly employed and are more likely to mixed feed than exclusively breastfeed in the first six months of their child's life [29]. 
CCPs may be influenced by a number of factors. One factor shown to have a significant association with the CCPs was the women' age. Women aged 35-44 are more likely $[\mathrm{OR}=1.65,95 \%$ (C. I: $0.56-4.88)]$ to use CCPs than those in the reference group (15-24 years) while women aged $\geq 45$ years are more likely $[\mathrm{OR}=0.58,95 \%$ (C. I: $0.18-1.88)]$. The finding is consonance with the finding of a study conducted which addressed the relationship between maternal age and infant feeding practices. The authors reported that older women are more likely to choose breastfeeding as their preferred infant feeding method [21]. However, this finding contradicts the findings of a survey that reported that no relationship was found between maternal age and feeding practices, though the percentage of respondents who chose to exclusively breastfeed increased with age [30].

Education enables people to appreciate the benefits that can be gained from complying with a recommended health intervention/regimen and can influence the overall health seeking behaviour of women. In this study, women with tertiary education are more likely $[\mathrm{OR}=2.45,95 \%$ (C. I: 1.01 - 5.94)] to use CCPs than those with no formal education. Furthermore, women with secondary education are less likely $[\mathrm{OR}=1.79,95 \%$ (C. I: $0.72-4.49)]$ to use CCPs than those with primary education where women in the primary, secondary education and tertiary education groups are associated with the use of CCPs at $\mathrm{P}<0.05$. The finding is anticipated because evidence abounds in literature that establish a strong correlation between maternal education and child care practices as such breastfeeding and uptake of childhood immunization services. Thus, women with no formal education will be hampered in their capacity to effectively take of their children due to educational limitations. This finding is consonance with findings of a study that reported that mothers with less education (high school diploma only or less) were more likely to rely on relative care than mothers with higher levels of education [29].

\section{Limitations of the Study}

This study has some limitations. Since it was a questionnaire-based, the responses may not have reflected the true opinions of all the respondents. Again since some of the respondents were not educated, there might have been diverse comprehension of the questions by such respondents following interpretations to the local language by the investigators. The study was designed to analyze factors associated with CCPs in Dekina LGA, north-central, Nigeria and may not be representative of the whole of Nigeria with its diverse sociocultural and demographic settings.

\section{Conclusion}

This study contributes to the limited knowledge base concerning factors associated with child care practices among mothers. As hypothesized by ecological theory [31], the systematic examination of individual, neighborhood, and other factors indicates that all may have important influences on CCPs by women. Symbolic interaction, one of the theories informing this study, emphasizes the influence of reference groups and significant others' opinions as shaping the self's choice of meaningful lines of action. This study to a very large extent confirms this theory as mothers were influenced by such groups in their selection of CCPs.

\section{Recommendations}

The following recommendations were in line with the study findings. These include:

1. Health workers and health authorities therefore are called upon to not only target the mother as the sole recipient of health education on child care practices, but also the general public, since anyone could be a reference point for mothers.

2. Mothers should also visit the primary health centres regularly in order to curb the initiation or mitigate the effects of any inappropriate child care practices.

3. There should a synergistic relationship between community health workers and mothers in order to facilitate efficient and effective communication channel. This can be used to inform mothers on current trend in child care practices. It can also serve as platform for feedback mechanism, and evaluation/assessment of women's progress and improvement on child care practices.

4. Holistic approach to understating factors affecting women's child care practices should be adopted because it will spur more research on CCPs. This may help reduce children's exposure to poor child care practices.

\section{Acknowledgements}

The authors would like to express sincere gratitude to medical officers (MOs) of sampled health facilities, health workers and women who gave their time and responses during the study, research assistants (nurses) who assisted during administration of copies of questionnaire at the sampled Hfs for their immense assistance and contributions.

\section{References}

[1] World Health Organization (2013). America's children and the environment: Measures of contaminants, body burdens, and illnesses (Report No 240-R-03-001). Washington DC. https://www.epa.gov/sites/production/files/201506/documents/ace3_2013.pdf Accessed August 14, 2015.

[2] World Bank (2012). Better health for our children: A national strategy (Volume III). Washington DC: Author: Select Panel for the Promotion of Child Health a Statistical Profile.

[3] Federal Ministry of Health (2010). National Strategic Health Development Plan (NSHDP) 2010-2015. Abuja, Nigeria: FMOH. Retrieved from: http: //www.health.gov.ng/doc/NSHDP.pdf. July, 2016. 
[4] Federal Ministry of Health, Nigeria (2012). Saving Newborn Lives in Nigeria: Newborn Health in the Context of the Integrated Maternal, Newborn and Child Health Strategy. Abuja, Nigeria: FMOH. Retrieved from http://countdown2015mnch.org/documents/countdownnews/nigeria-full-report.pdf July 15, 2016

[5] Tukur, J., Ahonsi, B., Ishaku, S. M., Araoyinbo, I., Okereke, E., \& Babatunde, A. O. (2013). Maternal and fetal outcomes after introduction of magnesium sulphate for treatment of preeclampsia and eclampsia in selected secondary facilities: A low-cost intervention. Maternal Child Health Journal, 17 (7), 1191-1198.

[6] Ushie, B. A., Fayehun, O. A., \& Ugal, D. B. (2014). Trends and patterns of under-5 vaccination in Nigeria, what manner of progress? Child Care Health Development, 40 (2), 267274.

[7] Findley, S. E., Doctor, H. V., Ashir, G. M., Kana, M. A., Mani, A. S., Green, C., et al. (2015). Reinvigorating health systems and community-based services to improve maternal health outcomes: Case study from Northern Nigeria. Journal of Primary Care Community Health, 6 (2), 88-99.

[8] United Nations Population Fund-UNPFA (2012, July). Key national indicators of well-being. Washington, DC: U.S. Government Printing Office: Health Resources and Services Administration Information Center.

[9] Balls, W. G. (2013). Origins and consequences of age at first drink. II: Familial risks and heritability. Alcoholism Clinical and Experimental Research, 25 (8), 1166-1173.

[10] Abel, E. L. (2010). Incidence of fetal alcohol syndrome and economic impact of FAS related anomalies. Drug and Alcohol Dependence, 19 (1), 51-70.

[11] National Research Council and Institute of Medicine (2004). Children's health, the nation's wealth: Assessing and improving child health. Washington, DC: NPA.

[12] Sanders, M. (2009). Infant feeding practices of low-income, African-American adolescent mothers: An ecological, multigenerational perspective. Social Science and Medicine, 49, 1085-1100.

[13] United Nations Children's Fund (2011). The effects of poverty on children and youth. Future of children. 55-71.

[14] Huston, A. C., Chang, Y. E., \& Gennetian, L. (2002). Family and individual predictors of child care use by low-income families in different policy contexts. Early Childhood Research Quarterly, 17, 441-469.

[15] Anderson, S. G., Ramsburg, D. M., \& Scott, J. (2005). Illinois study of license-exempt child care: Final report. Washington, D. C.: U. S. Department of Health and Human Services. Administration on Children, Youth, and Families, Child Care Bureau. Retrieved from www.researchconnections.org/location/ccrca7350

[16] Center for Community Child Health (2004). The underlying factors affecting child health, development and family functioning. Australia, Melbourne: Center for Community Child Health, Royal Children's Hospital, Melbourne. pp. 1-21.
[17] Hornby, A. S. (2001). Oxford advanced learners' dictionary of English ( $6^{\text {th }} \mathrm{ed}$.). New York: Oxford University Press.

[18] UNICEF (2005). State of the world's children 2005- childhood under threat. New York: UNICEF.

[19] Ortiz, I., Daniels, L. M., \& Engilbertsdóttir, S. (Eds.) (2012). Child poverty and inequality new perspectives. New York: United Nations Children's Fund, Division of Policy and Practice.

[20] Newman, J., \& Pittman, T. (2000). The ultimate breastfeeding book of answers. New York, NY: Three Rivers Press.

[21] Scott, J. A., \& Mostyn, T. (2003). Women's experiences of breastfeeding in a bottle-feeding culture. Journal of Human Lactation, 19 (3), 270-277.

[22] Irwin, L. G., Siddiqi, A., \& Hertzman, A. (2007). Early child development: A powerful equalizer. Final report of the Early Child Development Knowledge Network. Geneva: World Health Organization. Retrieved from http://www.who.int/social_determinants/publications/earlychil ddevelopment/en/index.html

[23] Borg, W. R. (1963). Educational research: An introduction. London: Longman.

[24] Akinware, M. A., \& Ojomo, A. A. (1993). Childrearing practices and their associated beliefs in Nigeria: A paper based on the baseline studies conducted in five Local Government Areas in Nigeria. UNICEF (1987-1990). Paper presented at the Workshop on Childrearing Practices and Beliefs, Windhoek, Namibia, October 26-29.

[25] Pungello, E. P., \& Kurtz-Costes, B. (2000). Working women's selection of care for their infants: A prospective study. Family Relations, 49 (3), 245-255.

[26] De Marco, A. C. (2006). Child care options and choices for families on welfare in rural northern California counties. Unpublished dissertation, UCLA, Berkeley.

[27] Kulkarni, M., \& Nimbalkar, M. (2008). Influence of sociodemographic factors on the use of antenatal care. Indian Journal of Preventive Social Medicine, 39 (3 \& 4), 98-102.

[28] Regassa, N. (2011). Antenatal and postnatal care service utilization in southern Ethiopia: A population-based study. African Health Science, 11 (3), 390-397.

[29] Bick, D. E., MacArthur, C., \& Lancashire, R. J. (1998). What influences the uptake and early cessation of breastfeeding? Midwifery, 14 (4), 242-247.

[30] Sika-Bright, S. (2010). Socio-cultural factors influencing infant feeding practices of mothers attending welfare clinic in Cape Coast, Ghana. Small Grants Programme in the Humanities and Social Sciences by the French Embassy, Accra, Ghana.

[31] Bronfenbrenner, U. (1994). Ecological model of human development. In International Encyclopedia of Education, Vol. 3, 2nd. Ed. In M. Gauvain, \& M. Cole, (Eds), Readings on the development of children, 2nd Ed. (1993, pp. 37-43). Oxford: Elsevier. 